\title{
TOPOLOGICAL GROUPS IN WHICH MULTIPLICATION OF ONE SIDE IS DIFFERENTIABLE
}

\author{
I. E. SEGAL
}

The present note is concerned with the problem of determining the formally weakest conditions on a group which can be proved to result in the group being a Lie group. We show essentially that it is sufficient for this purpose to require that the group be a (real) manifold of class $C^{1}$ in which right multiplication is of class $C^{1}$ (our result is slightly stronger-Theorem 2 is the precise statement). The weakest previously existing condition is contained in work of $\mathrm{P}$. A. Smith [2], ${ }^{1}$ and required, in addition to the preceding condition, that left multiplication satisfy a Lipschitz condition. ${ }^{2}$

We should point out that our result is obtained by combining previous work on Lie groups with a theorem which we prove independently of previous work. Specifically, we first prove that if right multiplication is of class $C^{1}$ on a group $G$ which is a manifold of class $C^{1}$, then it follows that left multiplication is of class $C^{1}$ (Theorem 1). We then deduce that $G$ is a Lie group either from the result of Smith quoted above, or from the result of G. Birkhoff [1] that if both left and right multiplication are of class $C^{1}$, then $G$ is a Lie group. We do not introduce any kind of canonical parameters, and, so far as we know, neither the work of Smith nor that of Birkhoff can be simplified by the use of our results.

Our method is novel in that it utilizes the existence of Haar measure and operates in the large. (Smith and Birkhoff were both concerned exclusively with the theory in the small, a standpoint incompatible with the use of Haar measure.) In this way it is shown that a wide collection of functions of $x$ of class $C^{1}, x$ being a variable group element, are also of class $C^{1}$ as functions of $x^{-1}$, and most of the proof is occupied with showing that this collection is sufficiently wide to allow the conclusion to be drawn that $x^{-1}$ is of class $C^{1}$ as a function of $x$. It follows that left multiplication is of class $C^{1}$. Only minor use is made of the theory of differential equations.

We are indebted to $\mathrm{D}$. Montgomery for highly suggestive conversations about the foundations of the theory of Lie groups.

The following theorem is valid for functions and manifolds of class

\footnotetext{
Presented to the Society, February 23, 1946; received by the editors January 19, 1946.

1 Numbers in brackets refer to the references cited at the end of the paper.

2 Smith had suggested that the Lipschitz condition might be inessential.
} 
$C^{k}(k \geqq 1)$ with an almost unchanged proof, but we state it for the case $k=1$ only.

THEOREM 1. Let $G$ be a topological group which is a manifold of class $C^{1}$, and such that $x a$ as a function of $x$ on $G$ is of class $C^{1}$, for every fixed $a$ in $G$. Then ax is likewise of class $C^{1}$.

Suppose that $f$ and $g$ are (real-valued) functions of class $C^{1}$ on $G$ which vanish except on a compact set. Then it is clear that the function $h$ defined by:

$$
h(x)=\int_{G} f(x y) g(y) d y
$$

is also of class $C^{1}, d y$ being left-invariant Haar measure, and it is easy to see that

$$
h\left(x^{-1}\right)=\int_{G} g(x y) f(y) d y,
$$

showing that $h\left(x^{-1}\right)$ is of class $C^{1}$ as a function of $x$, also. It is first shown (Lemma 1), using properties of Haar measure, that the collection of functions having the form (1) has the property that for any two given points of the group there exists a function in the collection which assumes different values at the points. It then follows (Lemma 2 ), by a method applicable to a general manifold, that $x^{-1}$ is of class $C^{1}$ as a function of $x$. Noting that:

$$
a x=\left(x^{-1} a^{-1}\right)^{-1},
$$

the conclusion follows from the fact that functional composition of functions of class $C^{1}$ results in a function of class $C^{1}$.

Since $x a$ is a function of $x$ of class $C^{1}$ we lose no generality by assuming now that if $(x \mid y)^{i}$ denotes the $i$ th coordinate of the point $x$ with respect to the local system at $y(i=1, \cdots, r)$, then $(x \mid y)^{i}=\left(x y^{-1} \mid e\right)^{i}$; here $e$ is the identity in $G, x$ and $y$ are elements of $G$, and $r$ is the dimension of $G$. We also assume that $(e \mid e)^{i}=0$.

LEMMA 1. Let $\Gamma$ be the collection of functions on $G$ of the form $\int_{G} f(x y) g(y) d y$, where $f$ and $g$ are real-valued functions on $G$ of class $C^{1}$, which vanish except on compact sets. Then for every two points of $G$ there exists an element of $\Gamma$ which assumes different values at the points. ${ }^{3}$

The proof is indirect: suppose that $\gamma(a)=\gamma(b)$ for all $\gamma \in \Gamma$, where

${ }^{3}$ We are indebted to $\mathrm{C}$. Chevalley and S. Eilenberg for suggesting a modification in the proof of the lemma which resulted in a considerably more elementary proof. 
$a \neq b$. Clearly, if $h(x)$ is a function of class $C^{1}$ on $G$, then the same is true of the function $h(x a)$, for every fixed $a$ in $G$. It follows that

$$
\int_{G} f(a y) g(y x) d y=\int_{G} f(b y) g(y x) d y,
$$

where $f$ and $g$ are general functions of class $C^{1}$ vanishing except on compact sets. Putting $h_{p}$ and $\bar{h}$ for the functions defined respectively by $\left(h_{p}\right)(x)=(h)\left(p^{-1} x\right)$ and $(\bar{h})(x)=\rho(x)(h)\left(x^{-1}\right)$, where $d x^{-1}=\rho(x) d x$, (3) can be written in the following form:

$$
\bar{f} * g_{a}=\bar{f} * g_{b} .
$$

Here $r * s$, for general complex-valued functions $r$ and $s$, denotes the function defined by:

$$
(r * s)(x)=\int_{G} r(y) s\left(y^{-1} x\right) d y,
$$

provided the integral exists. We now show that (4) is valid if $f$ and $g$ are integrable functions vanishing outside of any right translations of a certain neighborhood $R$ of the identity.

Specifically, let $P$ be the neighborhood of the identity defined by the inequalities $\left|(x \mid e)^{i}\right|<a(i=1, \cdots, r)$, where $a$ is chosen suffciently small so that the closure of $P$ is compact; let $Q$ be the same with $a$ replaced by $a / 2$, and let $R$ be a neighborhood of the identity such that $R^{2} \subset Q$. If $h$ is a continuous function which vanishes outside of $Q d$, then, putting $(x \mid d)^{i}=x$, and $h *\left(x_{1}, \cdots, x_{r}\right)=h(x)$ in the present sentence, we define $h_{\bullet}$ by

$$
h_{e}(x)=(2 e)^{-n} \int_{x_{r}-\bullet}^{x_{r}+\bullet} \cdots \int_{x_{1}-\bullet}^{x_{1}+\bullet} h_{*}\left(t_{1}, \cdots, t_{r}\right) d t_{1}, \cdots, d t_{r} .
$$

It is clear that if $0<e<1 / 2 a, h_{\theta}$ is defined, of class $C^{1}$, and converges uniformly to $h$ on $P d$ as $e \rightarrow 0$. If we set $\|r\|$ for the $L_{1}$ norm of $r$ (that is, the integral of the absolute value of $r$ with respect to Haar measure), it is plain that $\left\|h_{e}-h\right\| \rightarrow 0$ as $e \rightarrow 0$. Since the operators $h \rightarrow h_{p}$ and $h \rightarrow \bar{h}$ are linear and isometric on $L_{1}$, it follows that (3) is valid if $f$ and $g$ are continuous and vanish outside right translations of $Q$. Now if $h$ is integrable and vanishes outside $R d$, and if $u_{S}(x)=(m(S))^{-1} f_{S}(x)$, where $m$ is Haar measure, $S$ is a neighborhood of the identity, and $f_{S}$ is the characteristic function of $S$, it is easy to verify that $u_{S *} h$ vanishes outside $S R d$. On the other hand, for any positive number $e$ there exists such an $S$ contained in $R$ and such that $\left\|u_{S^{*}} h-h\right\|<e$. It follows that (3) is valid if $f$ and $g$ are integrable and vanish outside a right translation of $R$. 
Now let $T$ be a neighborhood of the identity such that $T=T^{-1}$, $T \subset R, a^{-1} T a \subset R$, and $a^{-1} b$ is not contained in $T^{3}$. Let $f$ and $g$ be the characteristic functions of $T$ and $a^{-1} T$ respectively. It is easy to verify that:

$$
\begin{aligned}
& \left(\bar{f}_{*} * g_{a}\right)(x)=m\left(a^{-1} T \cap a^{-1} T x^{-1}\right), \\
& \left(\bar{f}^{*} g_{b}\right)(x)=m\left(b^{-1} T \cap a^{-1} T x^{-1}\right) .
\end{aligned}
$$

Clearly $\bar{f}_{*} g_{a}$ and $\bar{f} * g_{b}$ are continuous functions, and it is easy to see that the first function is not zero at the identity and that the second function vanishes in $T$. Hence $\bar{f} * g_{a} \neq \bar{f} * g_{b}$, a contradiction. ${ }^{*}$

Lemma 2. Let $M$ be a manifold of class $C^{1}$ and dimension r. Let $\Phi$ be a collection of functions of class $C^{1}$ on $M$, with the property that for any two given points of $M$ there exists a function in $\Phi$ which assumes different values at the points. Then there exists a point of $M$ and $r$ elements of $\Phi$ whose Jacobian wrth respect to local coordinates at $p$, evaluated at $p$, is not zero.

Again our proof is indirect: assume that the Jacobian of any $r$ elements of $\Phi$ with respect to local coordinates at $x$, evaluated at $x$, vanishes for every $x \in M$. Let $s$ be the maximum rank of the matrices of all such Jacobians, let $\bar{x}$ be a point at which the rank is attained, and let $f_{1}, \cdots, f_{s}$ be elements of $\Phi$ involved in a nonvanishing minor of order $s$ in a Jacobian matrix at $\bar{x}$. For the remainder of the lemma, put $x_{i}=(x \mid \bar{x})^{i}$, and then re-index the coordinates so that $x_{1}, \cdots, x_{\text {, }}$ are the coordinates involved in the nonvanishing minor just mentioned. Then bordering this minor by adding any $f \in \Phi$ and any local coordinate around $\bar{x}$ not yet involved in the minor, say $x_{s+1}$, the resulting minor of order $s+1$ vanishes in a neighborhood of $\bar{x}$, clearly. Expanding the minor as a sum of products of row elements involving $f$ with the corresponding cofactors, we obtain an equation of the form

$$
\sum_{j=1}^{r} \frac{\partial f}{\partial x_{j}} A_{j}\left(x_{1}, \cdots, x_{r}\right)=0,
$$

where the $A_{j}$ are continuous functions and independent of $f$, with $A_{j}=0$ for $j>s+1$, and $A_{s+1}(0, \cdots, 0) \neq 0$. Now let $\left\{x_{i}(t)\right\}$ be a solution in a neighborhood of $t=0$ of the system of equations:

$$
\frac{d x_{i}}{d t}=A_{i}\left(x_{1}, \cdots, x_{r}\right), \quad x_{i}(0)=0 \quad(i=1, \cdots, r) .
$$

\footnotetext{
I It is interesting to note that the set of functions of class $C^{1}$ which vanish outside compact sets is $L_{1}$-dense in $L_{1}$. This fact could be proved, and the proof of the lemma shortened, by using theorems due to $\mathrm{H}$. Whitney.
} 
Plainly, in no neighborhood of $t=0$ do all $x_{i}(t)$ vanish identically. On the other hand, it is clear from the partial differential equation in $f$ that $f(x(t))=f(x)$, where $x(t)$ is the point whose coordinates are $\left(x_{1}(t), \cdots, x_{r}(t)\right)$, for all $t$ in some neighborhood of $t=0$. This equation clearly contradicts the hypothesis that $\Phi$ contains a function assuming different values at any two given points.

To conclude the proof of Theorem 1, let $M$ be an arbitrary open set in $G$, set $\Gamma=\Phi$, and let $p$ be a point of $M$ at which there exist $r$ elements $\left\{\phi_{i}\right\}$ of $\Gamma$ which have a nonvanishing Jacobian; by Lemmas 1 and $2, p$ and $\left\{\phi_{i}\right\}$ exist, evidently. By the theory of implicit functions, $\left\{(x \mid p)^{i}\right\}$ is a function of $\left\{\phi_{i}(x)\right\}$ of class $C^{1}$ in a neighborhood of $p$, say

$$
(x \mid a)^{i}=F_{i}\left(\phi_{1}(x), \cdots, \phi_{r}(x)\right)
$$

$$
(i=1, \cdots, r)
$$

Putting $x=y^{-1}, a=b^{-1}$, (5) becomes:

$$
\left(y^{-1} \mid b^{-1}\right)^{i}=F_{i}\left(\phi_{i}\left(y^{-1}\right), \cdots, \phi_{r}\left(y^{-1}\right)\right) \quad(i=1, \cdots, r) .
$$

Recalling that $\phi_{i}\left(y^{-1}\right)$ is of class $C^{1}$ as a function of $y$, it is clear from (6) that $y^{-1}$ is of class $C^{1}$ as a function of $y$, for $y$ in a neighborhood of $b$. Since $M$ is an arbitrary open set, the set $S$ of points at which there exists a set of $r$ elements of $\Phi$ with a nonvanishing Jacobian is dense in $G$; hence the same is true of the set $S^{-1}$, and therefore $G$ is covered by neighborhoods in which $y^{-1}$ is of class $C^{1}$ as a function of $y$.

Theorem 1 (which is a result in the large) is now combined with an existing result in the theory of local Lie groups, to obtain a condition that a group be a Lie group. At the same time we show that in order for right multiplication to be of class $C^{1}$ on a group which is a manifold of class $C^{1}$, it is sufficient for the first-order derivatives of right multiplication to exist, and be continuous at one point.

Theorem 2. Let $G$ be a connected topological group. Let there exist a neighborhood $N$ of the identity in $G$ which is a manifold of class $C^{1}$ and dimension $r$, and a neighborhood of the identity $P$ such that $P=P^{-1}$, $P^{2} \subset N$, and the first-order derivatives of $\left\{(x a \mid y a)^{i} ; i=1, \cdots, r\right\}$ with respect to $\left\{(x \mid y)^{i} ; i=1, \cdots, r\right\}$ exist for every fixed $a$ and $y$ in $P$, at $x=y$, and for one value of $y$ and all values of $a$ in $P$ are continuous; here $\left\{(x \mid y)^{i} ; i=1, \cdots, r\right\}$ are the local coordinates at $y$ of $x$. Then $G$ is a Lie group.

Let $f(s)$ be a real-valued function whose derivative (by derivative we mean first-order derivative in the following) exists for $s$ in an interval which includes the range of values of the real-valued function $g(t)$, as $t$ ranges over an interval containing the point $t_{0}$ in its 
interior; assume also that the derivative of $g(t)$ exists for $t$ in such an interval, and that the derivative of $f(s)$ is continuous at $s=g\left(t_{0}\right)$. Then it is easy to conclude from the mean value theorem of the differential calculus that the derivative of $f(g(t))$ exists at $t=t_{0}$ and equals $f^{\prime}\left(g\left(t_{0}\right)\right) g^{\prime}\left(t_{0}\right)$. Now the identity transformation on $G$ can clearly be obtained by first performing the transformation $x \rightarrow x a^{-1}$, and then following by the transformation $x \rightarrow x a$. If we put $x_{0}$ for the point at which $x a$ has continuous derivatives as a function of $x$ for all $a$ in $P$, then the derivatives of the first transformation exist at $x=x_{0} a=v_{a}$, if $v_{a} \in P$; and the derivatives of the second transformation exist and are continuous at $x=x_{0}$. It follows that:

$$
I=\left[\frac{\partial\left(x a^{-1}\right)}{\partial(x)}\right]_{x=v_{a}} \cdot\left[\frac{\partial(x a)}{\partial(x)}\right]_{x=x_{0}},
$$

where $I$ is the identity matrix, and $[\partial(x c) / \partial(x)]_{x=y}$ stands for the Jacobian matrix of the function $x c$, with respect to local coordinates at $y$ and $y c$, evaluated at $x=y$. Since $[\partial(x a) / \partial(x)]_{x=u}$ is continuous at $u=x_{0}$, it follows that $\left[\partial\left(x a^{-1}\right) / \partial x\right]_{x=u}$ is continuous at $u=v_{a}$, if $v_{a} \in P$. That is, $x a^{-1}$ as a function of $x$ has continuous derivatives at $x=v_{a}$, if $v_{a} \in P$. Hence $x a^{-1} b$ as a function of $x$ has continuous derivatives at $x=v_{a}$, if $a, b$, and $v_{a}$ are all in $P$. Plainly, $x_{0} P$ is an open set containing $e$; therefore there exists a neighborhood $Q$ of $e$ such that $Q=Q^{-1}, Q \subset x_{0} P$. Let $R$ be a neighborhood of $e$ such that $R=R^{-1}$, and $R^{2} C Q$. It is plain that as $a$ ranges over $x_{0}^{-1} R$ (which is a subset of $P$ ), $v_{a}$ ranges over $R$, and it is easy to verify that if $a \in x_{0}^{-1} R$, then $a^{-1} P \supset R$. It follows that $x a$ is of class $C^{1}$ as a function of $x$, for $x$ and $a$ in $R$.

Now let $S$ be a cubical neighborhood of $e$ such that $S^{-1} S \subset R$. We now regard $G$ as a manifold with local coordinates $[x \mid a]^{i}$ defined to be $\left(x a^{-1} \mid e\right)^{i}(i=1, \cdots, r)$ in the neighborhood $S a$ of $a$. At the identity this system of coordinates is the same as the original system, in $S$, and we now show that $G$ is a manifold of class $C^{1}$. If the neighborhoods $S a_{1}$ and $S a_{2}$ overlap, it is easy to verify that $a_{2} a_{1}^{-1} \in R$. Since the coordinates of $x$ relative to the $S a_{j}$ system are $\left\{\left(x a_{j}^{-1} \mid e\right)^{i}\right.$; $i=1, \cdots, r\}$, with $x a_{j}^{-1} \in S$, and multiplication on the right by the element $a_{2} a_{1}^{-1}$ of $G$ is of class $C^{1}$ on $N$, it follows that the $\left[x \mid a_{1}\right]^{i}$ $(i=1, \cdots, r)$ are of class $C^{1}$ as functions of the $\left[x \mid a_{2}\right]^{i}(i=1, \cdots, r)$. Clearly, $x a$ is of class $C^{1}$ as a function of $x$, at all points of $G$ and for all fixed $a$.

Then the hypothesis of Theorem 1 is satisfied. If we combine the conclusion of the theorem either with Smith's Theorem 12.9 [2] or Birkhoff's Corollary 15.7 [1], it follows that $G$ is a Lie group. 


\section{REFERENCES}

1. G. Birkhoff, Analytical groups, Trans. Amer. Math. Soc. vol. 43 (1938) pp. 61101.

2. P. A. Smith, Foundations of the theory of Lie groups with real parameters, Ann. of Math. vol. 44 (1943) pp. 481-513.

3. O. Veblen and J. H. C. Whitehead, The foundations of differential geometry, Cambridge, England, 1932.

4. A. Weil, L'Intégration dans les groupes topologiques et ses applications, Actualités scientifiques et industrielles, no. 869, Paris, 1940.

Institute for Advanced Study 